\title{
Spherical Planetary Ball Transmissions: Geometrical Synthesis
}

\author{
Mikhail E. Lustenkov \\ Belorussian-Russian University, Mogilev, Belarus \\ Email: lustenkov@yandex.ru
}

Received 7 April 2014; revised 27 May 2014; accepted 6 June 2014

Copyright $@ 2014$ by author and OALib.

This work is licensed under the Creative Commons Attribution International License (CC BY). http://creativecommons.org/licenses/by/4.0/

c) (i) Open Access

\section{Abstract}

The paper examines theoretical problems of the development of transmissions with intermediate engaged rolling bodies in which speed reduction and load transfer are made through interaction of main gear units with balls. The distinctive feature of this type of gears is the execution of working surfaces (running ways) of the main gear units on the spherical surfaces. The design of spherical transmission with the constant reduction ratio is developed. The principle of its work is based on interaction of rolling bodies with running way of drive shaft, working surfaces of stopped gear unit and meridional grooves of driven shaft. The running way and working surfaces are made along the harmonic curves closed on sphere. The parametrical equations of interacting spherical curves of several types are received. One of these curves is an inclined circle along which a ring groove on sphere is made. Theoretical principles are illustrated by examples of design of reducer unit.

\section{Keywords}

Spherical Planetary Transmissions, Spherical Curve, Reduction Gear, Cam Mechanism

\section{Introduction}

Mechanical transmissions with intermediate engaged rolling bodies are being developed in many countries [1]. The type of transmissions in question has a number of advantages inherent in planetary and wave gears, such as compactness, multiflow, high overlap ratio. A partial change of sliding friction for rolling friction facilitates lowering of losses in engaging. Without competing with traditional gears over the whole spectrum of reduction gears of general engineering application, the transmissions in question, however, can solve definite problems in special fields of engineering practice.

Transmissions with radial [2] and axial [3] movements of rolling bodies in the process of work are well 
known. Some mechanisms contain many intermediate movable elements and are difficult to make and assemble [4]. Spherical transmissions are less investigated, however, due to their application there appear possibilities of improvement of such mechanisms as constant-velocity universal spherical joints, couplings, mechanisms of movement removal in eccentric gear with an additional pass of gearing etc.

Known spherical-cam mechanisms are applied in cases when, because of machine space limitation, the shaft axes may intersect each other [5]. As an example, a pith-roll wrist designed with spherical multilobe-cam mechanism is described in [6]. But these mechanisms carry out specific tasks and, as a rule, do not provide constant value of the reduction ratio.

The aim of this paper is to develop geometrical principles of the creation of such transmissions with constant reduction ratio and an increase in technological effectiveness of their separate transmission units.

\section{Geometrical Principles of the Development of Spherical Planetary Ball Gears}

Let us introduce the following notions: cylindrical curve, unfolding of the cylindrical curve on the plane, spherical curve. A cylindrical curve is a spatial, closed, period curve situated on the cylindrical surface. The recurrent change of coordinate $z$ takes place relative to the middle line-circumference situated in $x O y$-plane with the centre in point $O$ and with radius $R$ equal to the radius of the forming cylindrical surface. On the middle line lies the whole number of periods $Z$ of curve. The unfolding of the cylindrical curve is a plane curve the ordinate of which is determined by equation $z=f(x)$. The spherical curve, correspondingly, is a spatial, closed, period curve situated on the spherical surface.

The notion of a cylindrical sinusoid was introduced in [7]. The transition from the equation of the curve on the cylinder to the equation on the sphere is made in the following way.

Let the cylindrical curve be given by the system of parameter equations $x=x(t), y=y(t), z=z(t)$, where $t$ is a changing parameter from 0 to $2 \pi R$ fulfilling the function of the angular position. The amplitude $A$ of curve is equal to section $D C$ or the length of arc $D^{\prime} C$ (Figure 1).

The transformations of the cylindrical curve into the spherical one are made by means of angle $\lambda$ (Figure 1 ):

$$
\lambda(t)=\frac{z(t)}{R}
$$

Parameter equations of the spherical curve are:

$$
\begin{gathered}
z_{s f}(t)=R \sin \lambda=R \sin \left(\frac{z(t)}{R}\right) \\
x_{s f}(t)=R \cos \left(\frac{t}{R}\right) \cos \lambda=R \cos \left(\frac{t}{R}\right) \cos \left(\frac{z(t)}{R}\right) \\
y_{s f}(t)=R \sin \left(\frac{t}{R}\right) \cos \lambda=R \sin \left(\frac{t}{R}\right) \cos \left(\frac{z(t)}{R}\right)
\end{gathered}
$$

For the cylindrical sinusoid with equation $z=A \sin (Z x / R)$ which is the equation of unfolding on $x O z$-plane the parameter equations are:

$$
x=R \cos \left(\frac{t}{R}\right) ; y=R \sin \left(\frac{t}{R}\right) ; z=A \sin \left(\frac{Z t}{R}\right)
$$

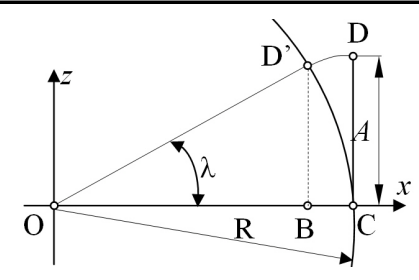

Figure 1. Scheme for transition from a cylindrical to a spherical curve. 
Consequently, parameter equations of the spherical sinusoid will look like:

$$
\begin{aligned}
& x_{s f}(t)=R \cos \left(\frac{t}{R}\right) \cos \left(\frac{A}{R} \sin \left(\frac{Z t}{R}\right)\right) \\
& y_{s f}(t)=R \sin \left(\frac{t}{R}\right) \cos \left(\frac{A}{R} \sin \left(\frac{Z t}{R}\right)\right) \\
& z_{s f}(t)=R \sin \left(\frac{A}{R} \sin \left(\frac{Z t}{R}\right)\right)
\end{aligned}
$$

The scheme of the mechanical transmission is shown in Figure 2, its running ways and working surfaces of transmission units being formed by Equations (6)-(8). The principle of its performance is based on the interaction of two curves (monoperiod and multiperiod), the middle lines of both curves coincide. Using curve data and with the help of the spherical milling cutter, running ways or working surfaces of two main transmission units contacting with rolling bodies (balls) are formed. The points of the intersection of these curves (not all but a definite group) are the points of the location of ball centers. Taken into consideration are only those points that are formed by the intersection of unlike curve branches: ascending and descending and vice versa. The total number of rolling bodies is equal to $b=Z_{1}+Z_{3}$, where $Z_{1}$ and $Z_{3}$ are period numbers of a monoperiod and multiperiod curve respectively.

It has been proved that the angular distance between the centers of any two balls when rotating relative to $z$-axis of one curve relative to the other is constant for cylindrical sinusoid [8], which enables a third main transmission unit to be introduced in the transmission construction, i.e. a shaft with slots situated along z-axis. It has been confirmed by us the preservation of the constancy of the angular distance for spherical surfaces by modeling in different CAD-programs [9].

The spherical planetary ball transmission (Figure 2) contains drive shaft 1 with a spherical internal cam having a closed running way. Coaxially to the internal cam are situated external crown cam 3 with a periodic surface and driven shaft 2 with slots on the internal spherical surface. These slots are located with a constant angular distance in the planes passing through the gear axis. The internal spherical surface of shaft 2 is also coaxial to cams 1 and 3. Rolling bodies 4 move along the running way of the internal cam on the drive shaft, on the working surfaces of the external cam and along the slots of the drive shaft forcing it to rotate with the reduction.

The external cam is rigidly connected with the gear casing (it is not shown in Figure 2) and the drive and driven shafts are situated in the casing (on bearing supports) with a console position of spherical surfaces. Transmissions of this type can be considered as cam mechanisms (combination of direct and indirect cam mechanisms), as a total amount of wedge mechanisms and as modification of wave gears where the system of rolling bodies forms a flexible deformed wave gear unit. Their belonging to the type of planetary gears is justified by the fact that their kinematics is completely subordinated to Willis' formula [10]. In this case the function of the number of teeth is fulfilled by the period numbers of curves, sizes of rolling bodies-satellites having no effect on the kinematic gear parameters.

\section{Synthesis of Transmission Geometry with a Running Way in the Form of a Circumference on the Internal Cam}

Let us consider a reverse side of the problem. Suppose there exists a spherical curve formed as a result of the plane trace passing through the origin of coordinates $O$ through one of the axes ( $x$ or $y$ ) and forming angle $\theta$ with the other axis (Figure 3). This trace will represent a circumference formed by turning of the middle (lying in $x O y$ plane) circumference-prototype with equations $\{x=R \cos (t / R), y=R \sin (t / R), z=0\}$. If plane inclination is made relative to $y$-axis, then as a result of turning there occurs the following transformation of the curve equations:

- since turning is made relative to $x$-axis, the transformation of coordinate $x$ does not take place, i.e. $x^{\prime}=R \cos (t / R)$;

- in $y O z$-plane section projection of $O M$ ( $M$ is an arbitrary point) onto $y$-axis makes turning to angle $\theta$. Let arbitrary point $M$ on the circumference-prototype have coordinates $x_{M}, y_{M}$, then after turning its coordinates will look like: $y_{M}^{\prime}=y_{M} \cos \theta=R \sin (t / R) \cos \theta, z_{M}^{\prime}=y_{M} \sin \theta=R \sin (t / R) \sin \theta$.

Thus, parameter equations of the curve formed as a plane trace on the spherical surface have the following form: 


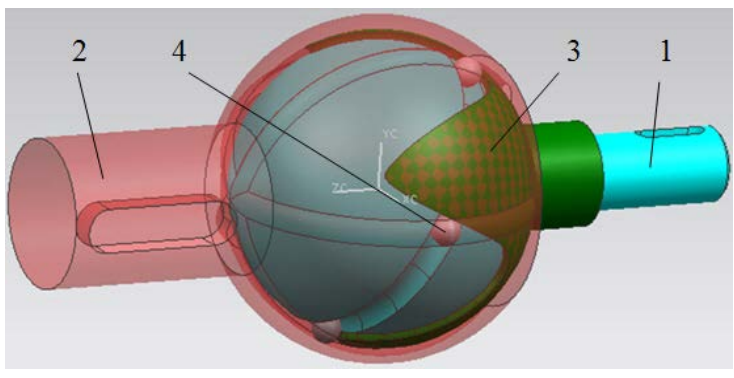

Figure 2. Spherical planetary ball transmission with sinusoidal curves.

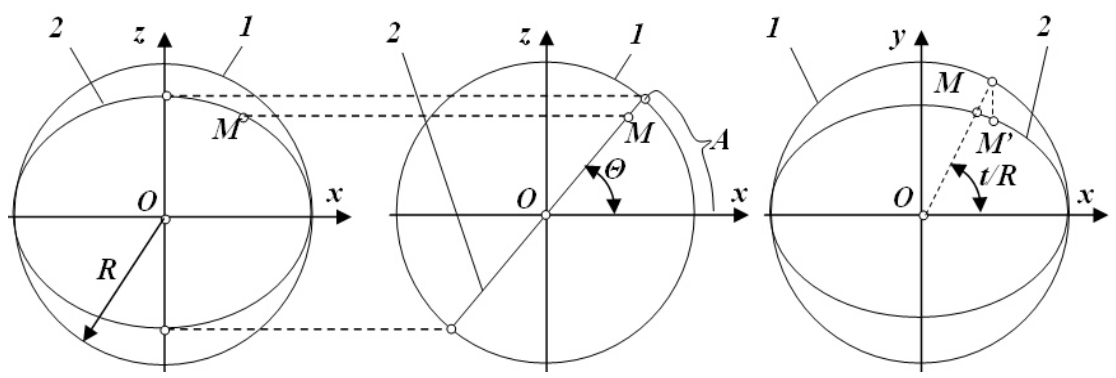

Figure 3. For determining of the equations of the plane trace on the sphere: 1 -spherical surface, 2 - plane trace.

$$
\begin{gathered}
x_{s f}=R \cos \left(\frac{t}{R}\right) \\
y_{s f}=R \sin \left(\frac{t}{R}\right) \cos \theta \\
z_{s f}=R \sin \left(\frac{t}{R}\right) \sin \theta
\end{gathered}
$$

Angle $\theta$ unlike angle $\lambda$ which depends on parameter $t$ (Figure 1 ) has a fixed value and equals to $\theta=A / R$.

Equations (9)-(11) are not equally comparable with Equations (2)-(4). The availability of multiperiod-coefficient $\cos \theta$ in Equation (10) leads to the determination of point $M$ coordinate (Figure 3) the projection of which onto $x O y$-plane does not lie on the ray outcoming from centre $O$ at angle $t / R$ to $x$-axis at the given value of parameter $t$. However, it is obvious that the circumference projection described by Equations (9)-(11) onto $x O y$ plane will represent on ellipse the equation of which is:

$$
\frac{x_{s f}^{2}}{R^{2}}+\frac{y_{s f}^{2}}{R^{2} \cos ^{2} \theta}=1
$$

Thus,

$$
x_{s f}=\frac{R \cos \theta \cdot \cos \left(\frac{t}{R}\right)}{\sqrt{\cos ^{2} \theta \cdot \cos ^{2}\left(\frac{t}{R}\right)+\sin ^{2}\left(\frac{t}{R}\right)}}
$$

Having expressed $y_{s f}$ from Equation (12) and having substituted $x_{s f}$ in it from the expression (13) and having made necessary transformations, we obtain: 


$$
y_{s f}=\frac{R \cos \theta \cdot \sin \left(\frac{t}{R}\right)}{\sqrt{\cos ^{2} \theta \cdot \cos ^{2}\left(\frac{t}{R}\right)+\sin ^{2}\left(\frac{t}{R}\right)}}
$$

Having substituted expression (13) and (14) in the sphere expression $x_{s f}^{2}+y_{s f}^{2}+z_{s f}^{2}=R^{2}$, we express coordinate value $z_{s}$ :

$$
z_{s f}=\frac{R \sin \theta \cdot \sin \left(\frac{t}{R}\right)}{\sqrt{\cos ^{2} \theta \cdot \cos ^{2}\left(\frac{t}{R}\right)+\sin ^{2}\left(\frac{t}{R}\right)}}
$$

Thus, parameter equations of the spherical curve can also be represented as expressions (13)-(15). If to consider this curve as a monoperiod one having transformed the above mentioned equations, we can obtain the equations describing the series of multiperiod curve of this type:

$$
\begin{gathered}
x_{s f}=\frac{R \cos \left(\frac{A}{R}\right) \cdot \cos \left(\frac{t}{R}\right)}{\sqrt{\cos ^{2}\left(\frac{A}{R}\right) \cdot \cos ^{2}\left(\frac{Z t}{R}\right)+\sin ^{2}\left(\frac{Z t}{R}\right)}} \\
y_{s f}=\frac{R \cos \left(\frac{A}{R}\right) \cdot \sin \left(\frac{t}{R}\right)}{\sqrt{\cos ^{2}\left(\frac{A}{R}\right) \cdot \cos ^{2}\left(\frac{Z t}{R}\right)+\sin ^{2}\left(\frac{Z t}{R}\right)}} \\
z_{s f}=\frac{R \sin \left(\frac{A}{R}\right) \cdot \sin \left(\frac{Z t}{R}\right)}{\sqrt{\cos ^{2}\left(\frac{A}{R}\right) \cdot \cos ^{2}\left(\frac{Z t}{R}\right)+\sin ^{2}\left(\frac{Z t}{R}\right)}}
\end{gathered}
$$

The interaction of two spherical curves mono- and 14-period is shown in Figure 4.

To obtain the equation of plane unfolding of the cylindrical curve it is necessary to make the following transformations according to Equation (2).

$$
z(t)=R \arcsin \left(\frac{z_{s f}(t)}{R}\right)=R \arcsin \left(\frac{\sin \left(\frac{A}{R}\right) \cdot \sin \left(\frac{Z t}{R}\right)}{\sqrt{\cos ^{2}\left(\frac{A}{R}\right) \cdot \cos ^{2}\left(\frac{Z t}{R}\right)+\sin ^{2}\left(\frac{Z t}{R}\right)}}\right)
$$

\section{Analysis of Synthesized Curves and the Development of Reduction Gear Construction}

Let us compare three types of monoperiod and multiperiod curves (Figure 5): sinusoid, section-screw curve and the curve obtained by Equation (19) at the same parameters: $A=10 \mathrm{~mm}, R=20 \mathrm{~mm}, Z=1$ and $Z=4$.

The section-screw curves on the sphere are described by parameter equations:

$$
\begin{gathered}
x_{s f}(t)=R \cos \left(\frac{t}{R}\right) \cos \left(\frac{2 A}{\pi R} \arcsin \left(\sin \left(\frac{Z t}{R}\right)\right)\right) \\
y_{s f}(t)=R \sin \left(\frac{t}{R}\right) \cos \left(\frac{2 A}{\pi R} \arcsin \left(\sin \left(\frac{Z t}{R}\right)\right)\right)
\end{gathered}
$$




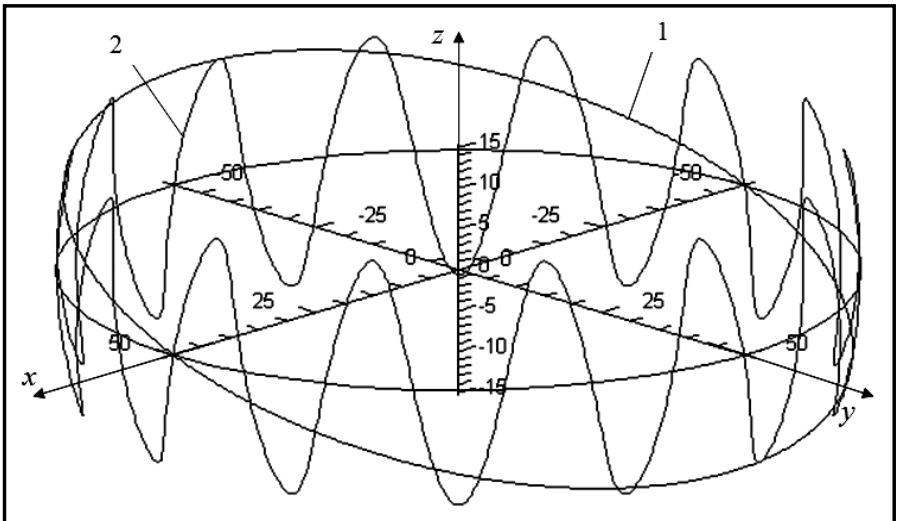

Figure 4. Spherical curves: 1-monoperiod, 2-14-period.

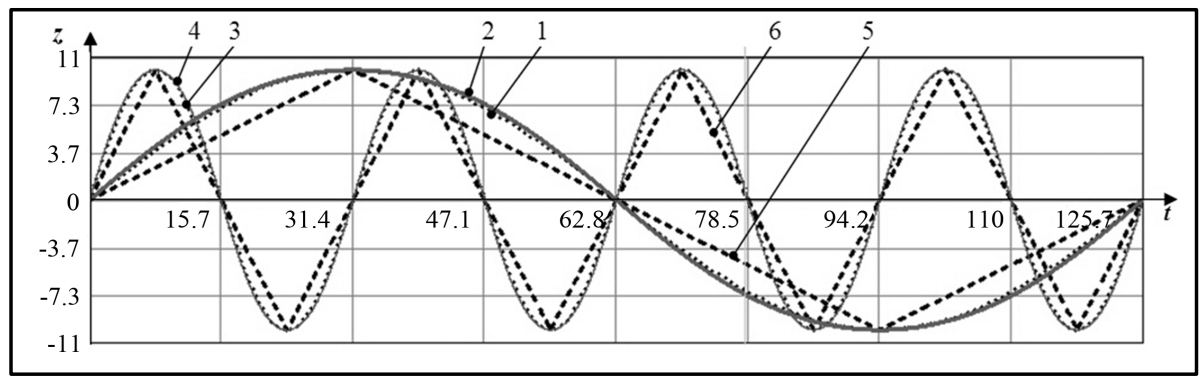

Figure 5. Unfoldings onto the plane of curves of different types: 1, 2-monoperiod sinusoid and curve on Equation (19); 2, 4-multiperiod sinusoid and curve on equation (19); 5, 6monoperiod and multiperiod section-screw curves respectively.

$$
z_{s f}(t)=R \sin \left(\frac{2 A}{\pi R} \arcsin \left(\sin \left(\frac{Z t}{R}\right)\right)\right)
$$

The equation of plane unfolding of the section-screw curve is:

$$
z=\frac{2 A}{\pi} \arcsin \left(\sin \left(\frac{Z t}{R}\right)\right)
$$

As you see from Figure 5, the difference between the sinusoid and the curve described by Equation (19) at the given parameters is actually indistinguishable. Unfolding onto the plane of the trace of plane section of the cylinder is a sinusoid, but a trace of plane section of sphere (with a plane which is passing through the center of it) is not a sinusoid. Figure 6 represents the dependence of changing the absolute value of the difference between coordinates $z$ of these curves on parameter $t$.

In order to exemplify the proposed synthesis method, a numerical example has been solved. Initially data: the reduction ratio is $u=15.0$, the maximal diameter of reducer is $D_{\max }=150 \mathrm{~mm}$. The factor of friction is accepted to be $f=0.05$ (motion with sliding and rolling). Thus it is considered, that the material of all details is hardened steel, factor of friction in all pairs is identical. The radius of a middle circumference can be calculated with empirical equation (from experience of designing of cylindrical ball transmissions): $R=(0.6 \cdots 0.8) D_{\max } / 2$. The diameter of a ball depends on its material, heat treatment and acting forces. Preliminary diameter of a ball can be calculated according to another empirical equation: $d_{b}=(0.05 \cdots 0.07) R$. Number of the periods of multiperiod curve is defined as $Z_{3}=u-1$. The optimal value of amplitude of curves (with maximum of efficiency) [11] is calculated with formula:

$$
A=\frac{\pi R \sqrt{2 Z_{3} Z_{1}-\tan ^{2} \psi}}{R}
$$

where $\psi$ is an angle of friction: $\psi=a \tan (f)$. 
The following values of parameters have been obtained: $R=50.0 \mathrm{~mm}, d_{b}=12.0 \mathrm{~mm}, Z_{3}=14, A=15 \mathrm{~mm}$.

The model of mechanism with spherical planetary ball transmission with reduction ratio 15.0 realized on the basis of the curve interaction described by Equations (20)-(22) is shown in Figure 7.

The difference of the reduction gear design in Figure 7 from that shown in Figure 2 is in the increase in technological effectiveness of the internal cam production. The running way of the reduction gear internal cam shown in Figure 7 is made in form of a circumference, but a monoperiod sinusoid (Figure 2) is more complex curve. The internal cam (Figure 7) is located on the shaft, the axis being inclined relative to $z$-axis at angle $\theta$. An angle of inclination of drive shaft will be $\Theta=A / R=15 / 50=0.3 \mathrm{rad}$. The inclined drive shaft is also in designs of transmissions with precession movement [12]. However, in these transmission there is an insignificant number of rolling bodies (teeth) in contact, and it reduces their loading ability.

Speed and acceleration of the centers of balls can be determined, having taken the first and the second time derivative of function $z(t)$. Parameter $t$ is equal to $(\omega R)$ )time (where $\omega$ is an angular velocity and its value is constant).

\section{Conclusions}

The given algorithms enable to design spherical planetary transmissions with interacting curves of different types. Each type of curves has its advantages and disadvantages. For example, it is expedient to use sinusoids at high rotation frequencies, since centre speeds of rolling bodies change under the harmonic law and at the curve apexes, where balls do not carry loads, they are equal to zero. Section-screw curves enable to reproduce a greater torque and have greater stability of the average value of the efficiency because of the constant slopes of

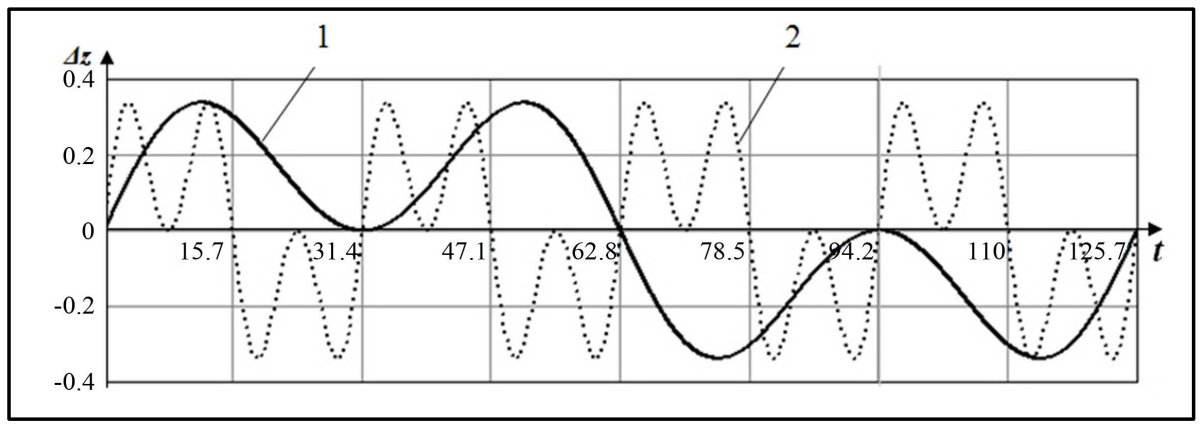

Figure 6. Difference in values of coordinate $z$ for sinusoids and curve on Equation (19): 1 -for monoperiod curves, 2 -for multiperiod curves.

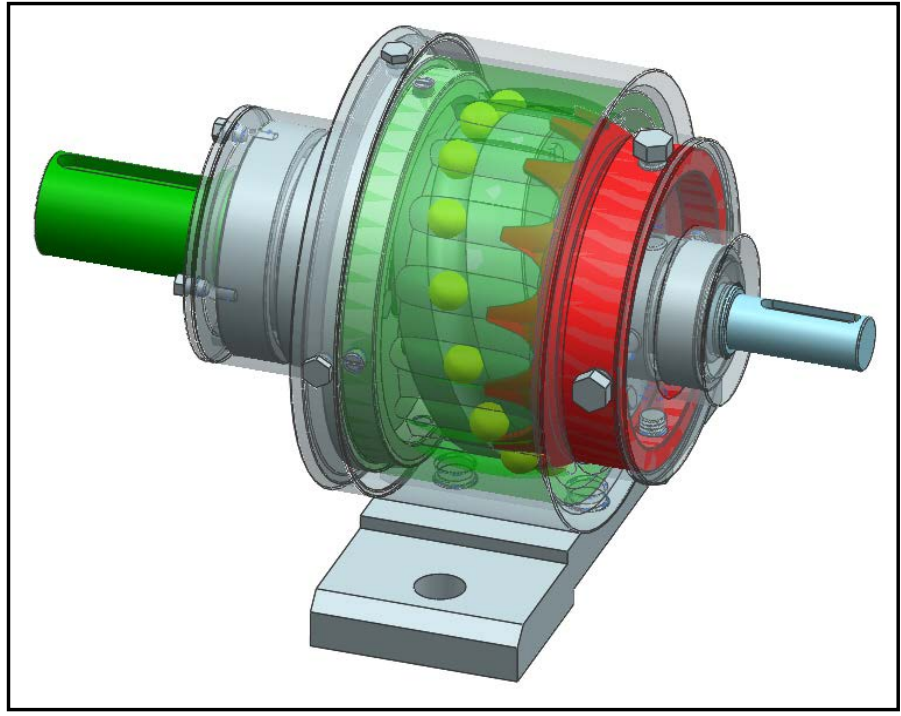

Figure 7. Spherical ball planetary reduction gear. 
interacting curves but impact between rolling bodies and working surfaces of gear units are possible at the apexes of these curves. The character of the curve interaction and the advantages that can be obtained when using curves of this or that type must be investigated by means of unfolding equations onto the plane, and, afterwards, these equations can be transformed into the parameter equations of cylindrical or spherical curves. Using the given algorithm one can synthesize the equations of multiperiod curves by the equations of monoperiod curves enabling to develop spherical planetary ball transmissions.

The universal joints can be built in the developed transmission, Rzeppa universal joint [13] for example. It will allow changing an inclination angle between axes of shafts of reducer unit during work. This building is easy to realize because both of the mechanisms have similar arrangement.

\section{References}

[1] Stanovskoi, V.V., Remneva, T.A. and Kazakyavichyus, S.M. (2003) Transmissions with Intermediate Rolling Bodies, Survey of the Patent Literature, Progressive Gearings. NGTI, Novouralsk, 61-94.

[2] Bara, M. and Brisan, C. (2004) Designing Aspects of a Special Transmission with Balls. Proceedings of the 8th World Congress in Mechanism and Machine Science, Tianjin, 1-4 April 2004, 872-878.

[3] Bara, M. (2006) Designing Aspects of Cylindrical Transmission. ASME Conference Proceedings, Chicago, 5-10 November 2006, 75-81.

[4] Terada, H., Masuda, T. and Yoshida, S. (2007) Motion Analysis of a Reciprocating Motion Type Ball Reducer. Proceedings of 12th IFToMM World Congress, Besançon, 18-21 June 2007, 414-418.

[5] Robert, O. and Parmley, P.E. (editor in chief) (2000) Illustrated Sourcebook of Mechanical Components. McCraw-Hill, New York.

[6] Bai, S. and Angeles, J. (2009) The Design of Spherical Multilobe-Cam Mechanisms. Proceedings of the Institution of Mechanical Engineers, Part C: Journal of Mechanical Engineering Science (London), 223, 473-482. http://dx.doi.org/10.1243/09544062JMES1154

[7] Ignatischev, R.M. (1983) Sinus-Ball Reducers. Higher School, Minsk.

[8] Lehmann, M. (1981) Sinoiden Getriebe. Konstruktion, B33, 109-112.

[9] Lustenkov, M.E. and Makarevich, D.M. (2005) Planetary Ball Transmissions of Cylindrical Type. Belorussian-Russian University, Mogilev.

[10] Willis, R. (1870) Principles of Mechanism. 2nd Edition, Longmans, Green and Co., London.

[11] Lustenkov, M.E. (2010) Transmissions with Intermediate Rolling Elements: Power Loss Estimation and Minimization. Belorussian-Russian University, Mogilev.

[12] Terada, H., Fukasawa, T. and Irie, R. (2003) Fundamental Analysis of a Precession Motion Ball Reducer (1st Report) -Motion Principle of a Coaxial Output Type. Journal of the Japan Society for Precision Engineering, 69, 688-692. http://dx.doi.org/10.2493/jispe.69.688

[13] Miller, F.F., Holzinger, D.W. and Wagner, E.R. (1979) Universal Joint and Drive Shaft Design Manual. 3.2.8 Rzeppa Universal Joint, SAE, Society of Automotive Engineers Inc., Warrendale, 145-150. 